\title{
Research on Construct of Energy Management Platform Based on Marketing and Distribution Integrated System
}

\author{
Bo $\mathrm{Hu}^{1,2}$ \\ ${ }^{1}$ State Grid Anshan Electric Power Supply Co. Ltd., Shenyang China \\ ${ }^{2}$ North China Electric Power University, Beijing China \\ * Corresponding author: hubosgccln@163.com
}

\begin{abstract}
Marketing and Distribution Integrated System is based on GIS systems, and a unified information model of power grid equipment, basic data and topology-based systems, the use of high-tech information technology, application management and distribution line losses quarter field of network construction planning, customer outage management and other integrated information platform. Energy management system implementation ultimate goal is to achieve through intelligent system integration of existing systems be saving energy consumption and improved. In automation technology and IT based on the establishment of energy management systems, based on objective data, metallurgical, chemical, thermal, power plants and other energy consuming enterprises, energy saving most fundamental way to implement. The foundation and core of this system is that the data distribution network GIS platform, integrated measurement automation, distribution network of production, distribution network planning, distribution network automation, distribution network engineering, marketing and other systems supporting the management and reliability of power supply and distribution network planning decisions Camp with information query formulated meter reading routes and other services.
\end{abstract}

Keywords: energy management platform, marketing and distribution, integrated system, GIS systems

\section{Introduction}

GIS (geographic information system) is developing rapidly in recent years, information systems, computer hardware and software in support of a variety of geographic information in accordance with the spatial distribution and properties to a certain format input, storage, retrieval, update, display, drawing, comprehensive analysis and application of technology systems [1-5]. Introduction of geographic information systems in the distribution automation system, according to the actual situation of the distribution network and the realization of the work requirements of the operation and management of distribution automation, electrical equipment and facilities information provided by the network operation status, power technical information, production management information, power market information and information about the actual geographical environment, urban construction and road traffic information by combining the use of databases and geographical information technology, grid space resources for effective collection and management, the establishment of practical maintenance tools electricity network resources, and this building electricity production, marketing and service of space resources information platform-camp with integrated information platform [6].

Energy management system is a very broad coverage of an integrated system, a number of technical fields related to building technology, industrial automation, data acquisition analysis. Energy management system implementation ultimate goal is to achieve through intelligent system integration of existing systems be saving energy consumption and improved [7]. In automation technology and IT based on the 
establishment of energy management systems, based on objective data, metallurgical, chemical, thermal, power plants and other energy consuming enterprises, energy saving most fundamental way to implement. Promote advanced energy management system application concepts. Change the traditional non-science-based energy production and management methods, is a modern large, medium and small businesses effective major advanced management measures, managers at all levels is becoming the consensus of major companies [8].

This article is in camp with the integration on the basis of the establishment of the basic purpose of the energy center management system is to improve the energy system in operation, management efficiency, energy consumption in the production process to find the best process data, providing a mature, effective, easy to use control overall energy system solutions; set of advanced, reliable and secure energy system operation, operation and management platform [9]. And to achieve security and stability, economic balance, the basic objectives of quality and environmental protection, supervision and evaluation. Through data analysis, can help enterprises each production line, each working team and the major energy-consuming equipment for real-time assessment, eliminate waste, and can help enterprises to further optimize the process in order to reduce energy consumption per unit costs and improve their overall competitiveness. For enterprise production management, measurement management, energy management to a new concept. Development and application of energy management system is our energy conservation, energy saving achieved an effective solution.

\section{Marketing and Distribution Integrated System}

Marketing and distribution integrated system is IT application integration project with the construction project is the use of modern information technology, on the basis of a unified enterprise network equipment and power users information model, basic information and topological relationship, applied to customer-facing supply reliability management, outage management, line loss quartile management, business expanding areas of decision-making and assist with network construction planning, standardization and integration of enterprise-class information platform [10]. By building an integrated digital grid, promote the construction of power supply enterprise resources and business information systems integration, integration of various discrete current production management system, to achieve the overall objective of a platform for information sharing, data only, integrated applications, production fusion between marketing, scheduling, engineering and other services, to specialization, refinement and standardization of enterprise management objectives.

\subsection{Disadvantages of Separation Mode in Marketing and Distribution}

Implementation of the "separation mode in marketing and distribution" of the mind, from the grassroots power grid companies and power grid operators to maintain the dual responsibility. The separation between the two, so $10 \mathrm{kV}$ distribution network maintenance more professional, more reliable grid operation stability can be achieved grid strong asset quality business strategy. This innovative management ideas, no doubt to the power companies how to run a business in the new situation, providing substantive significance reference value. But as a new thing, this mode of operation in the current process, there are still some problems, I believe that these problems are mainly embodied in the following four aspects.

According to the current "camp with separate" management mode, only the $10 \mathrm{kV}$ network portion of the work area was placed under the newly established distribution maintenance and management, while 400 volts and below the distribution network, is still owned by the seat of power assumed responsibility for the maintenance and management. In the distribution network maintenance work, the actual maintenance tasks should belong 
to the most important distribution network 400 volts and below its long lines, complex structure, failure and more.

After the reform, the power of the original personnel responsible for maintenance tasks, still not fully converted management personnel, but also need to keep the original repair maintenance organizations, safeguard routine maintenance management. Since the reform of supporting policies in place, some areas of supply enterprises according to their service characteristics, its own links with the local price departments, developed a standard of paid services, but most areas did not take paid services management measures in only emphasizes quality At the same service, there is no source of income for support, service funds are not included in operating costs, resulting in this work is difficult to maintain. Especially rural migrant workers who are currently increasing, many families lack electricity understand young people, who are left behind the old, small, small, really need to supply enterprises with professional maintenance personnel responsible for processing power failure, resolve thousands of families electricity problems. If the system of paid services, extension of electricity service range, grass-roots power of the maintenance personnel increased rather than decreased, the implementation of "camp with separation" of the mind, will be difficult to get reflected. But if the 400 volts and below the work area is responsible for the distribution and distribution networks into maintenance management, will significantly increase its maintenance costs, and can not be guaranteed to reach the fault location within the time promised, and will delay the optimal repair time, causing some serious accidents .

According to the basic settings "camp with separate" work 10kV line (with transformer low-voltage outlet side) the installation, maintenance, responsible for distribution work area, the primary power supply is no longer engaged in the above work, and the power supply are engaged in business indicators management, coordination of work in process, with particular attention to clear the configuration and installation of metering devices permission, otherwise it is easy to trouble the former to the latter's business management. Because, after "camp with separation," installation work area right by the distribution of all, its budget and configuration right, must have first-hand information provided by the local power distribution work area, if not available, installation time is not clear, it matching calculation will lead to unreasonable rate, power, electricity miscalculation large customer base tariff of the starting time of the occurrence of errors and other economic disputes.

\subsection{GIS Application in Power System}

GIS application in power system has touched all aspects of the power system. From transmission and distribution to customer service; equipment from everyday management, operational management, project management, defect management, reliability management to the line of the design, construction, planning, and so on.

Transmission grid is relatively simple, but cover a large geographical area, carried out earlier application of GIS systems. Not only been widely used in the operation and maintenance of the line, in terms of the degree of application of circuit design, planning, etc. are also increasing. Currently the vast majority of the provincial network and the State Grid Corporation and Southern regional power company owned network are established based on GIS transmission NMS.

Distribution network wiring complexity, frequent changes, network-intensive, but for the application of these features enhance the GIS system is extremely suitable space. At present, the capital city of the prefecture-level city of the State Grid Corporation and Southern Grid Corporation owned and developed areas, and even county-level city of power supply enterprise has established a distribution network management system based on GIS.

GIS systems such as power transmission and distribution not only in the production sector has been widely used in the future services also play an increasingly important role 
in other systems such as electricity marketing systems, customer service systems (voice systems, call Management system) to cooperate fully with, can provide more convenient services for power users, but also to shorten the outage time, improve reliability, improve service levels supply companies provide technical support.

\section{Energy Efficiency Management System Architecture and Operating Principle}

\subsection{System Architecture}

Energy management system architecture can be divided into the terminal from the device layer, a communication link layer, business layer and service support management, overall system architecture shown in Figure 1.

\begin{tabular}{|c|c|c|c|}
\hline \multicolumn{3}{|c|}{ Business support layer } & Service Management \\
\hline Data Integration & $\begin{array}{l}\text { Energy efficiency } \\
\text { program evaluation }\end{array}$ & $\begin{array}{l}\text { Energy efficiency } \\
\text { indicator evaluation }\end{array}$ & $\begin{array}{l}\text { Customer information } \\
\text { management }\end{array}$ \\
\hline $\begin{array}{l}\text { Saving potential } \\
\text { evaluation }\end{array}$ & $\begin{array}{l}\text { Customer energy- } \\
\text { saving evaluation }\end{array}$ & $\begin{array}{l}\text { After evaluating } \\
\text { energy-saving }\end{array}$ & \multirow{2}{*}{$\begin{array}{l}\text { Customer acquisition } \\
\text { and satisfaction } \\
\text { management }\end{array}$} \\
\hline & & & \\
\hline \multicolumn{3}{|c|}{ Communication link layer } & $\begin{array}{l}\text { Periodic evaluate } \\
\text { management tracking }\end{array}$ \\
\hline Error checking & $\begin{array}{l}\text { Signal } \\
\text { amplification }\end{array}$ & $\begin{array}{l}\text { Communication } \\
\text { protocol conversion }\end{array}$ & \multirow{2}{*}{$\begin{array}{l}\text { Fault repair } \\
\text { management }\end{array}$} \\
\hline Noise removal & Data Coding & $\begin{array}{l}\text { Link Lifecycle } \\
\text { Management }\end{array}$ & \\
\hline & & & $\begin{array}{l}\text { Training Services } \\
\text { Manager }\end{array}$ \\
\hline \multicolumn{3}{|c|}{ Terminal Equipment layer } & \multirow{2}{*}{ Product marketing } \\
\hline Power monitor & $\begin{array}{l}\text { Reactive power } \\
\text { compensation device }\end{array}$ & $\begin{array}{l}\text { New Energy } \\
\text { Equipment }\end{array}$ & \\
\hline Smart meter & Filtering device & $\begin{array}{l}\text { Protection } \\
\text { devices }\end{array}$ & Partner Development \\
\hline
\end{tabular}

Figure 1. System architecture

Terminal equipment level, mainly in the hardware level to achieve energy efficiency is optimized by a variety of intelligent installed at the customer site constitutes energy efficient equipment. Including power quality can be optimized devices (reactive power compensation devices, active and passive filter device, inverter, etc.), used to collect data to monitor a variety of advanced energy metering equipment (acquisition, concentrator, power monitor, etc.), low-carbon environmental protection and new energy equipment (photovoltaic systems, wind power systems, CCHP systems, etc.). Energy efficiency can actually among heterogeneous devices for the needs of customers in different industries and economic levels matching and optimization portfolio, reducing the overall cost of terminal equipment installation.

Communication link layer is responsible for the communication management data exchange, data transmission, etc. Including the link establishment and removal, monitoring and maintaining the link abnormal signal amplification and noise removal, data validation and error correction, data coding and communication protocol conversion functions, improve the terminal equipment to provide data reliability and availability. Business support layer is mainly achieved by the system software on the client load and energy data, industry-standard data, data analysis and management of power within the 
system to achieve energy efficiency targets grid management, customer energy efficiency potential assessment. Underlying business through massive data integration, real-time and offline data management, mathematical modeling and data analysis, to provide energysaving program evaluation, assessment of energy-saving potential, energy efficiency indicators to assess customer base multi-dimensional assessment of energy-saving programs, basic data from multiple angles. Business intelligence available to customers and the upper transparent user interface, through the formation of a complete set of customer interaction optimization of energy efficiency programs, and to achieve energy efficiency and energy efficiency to predict the assessment and other functions.

Services for energy management consulting services assist clients manage more effectively. Customer information management, customer acquisition and satisfaction of collecting and tracking assessment stage, mainly by getting customers to use feedback and data analysis to help end customers can demand correction, custom personalized energy-saving programs. Fault repair management and training mainly to provide after sales service management technology and operation and maintenance support. In addition, through product marketing, development partners to further expand the coverage of energy efficiency services, improve the degree of audience.

\subsection{System Software Architecture}

Energy management system software is structured into the system platform layer, the data support layer, the public component layer and application layer model, system software structure shown in Figure 2.

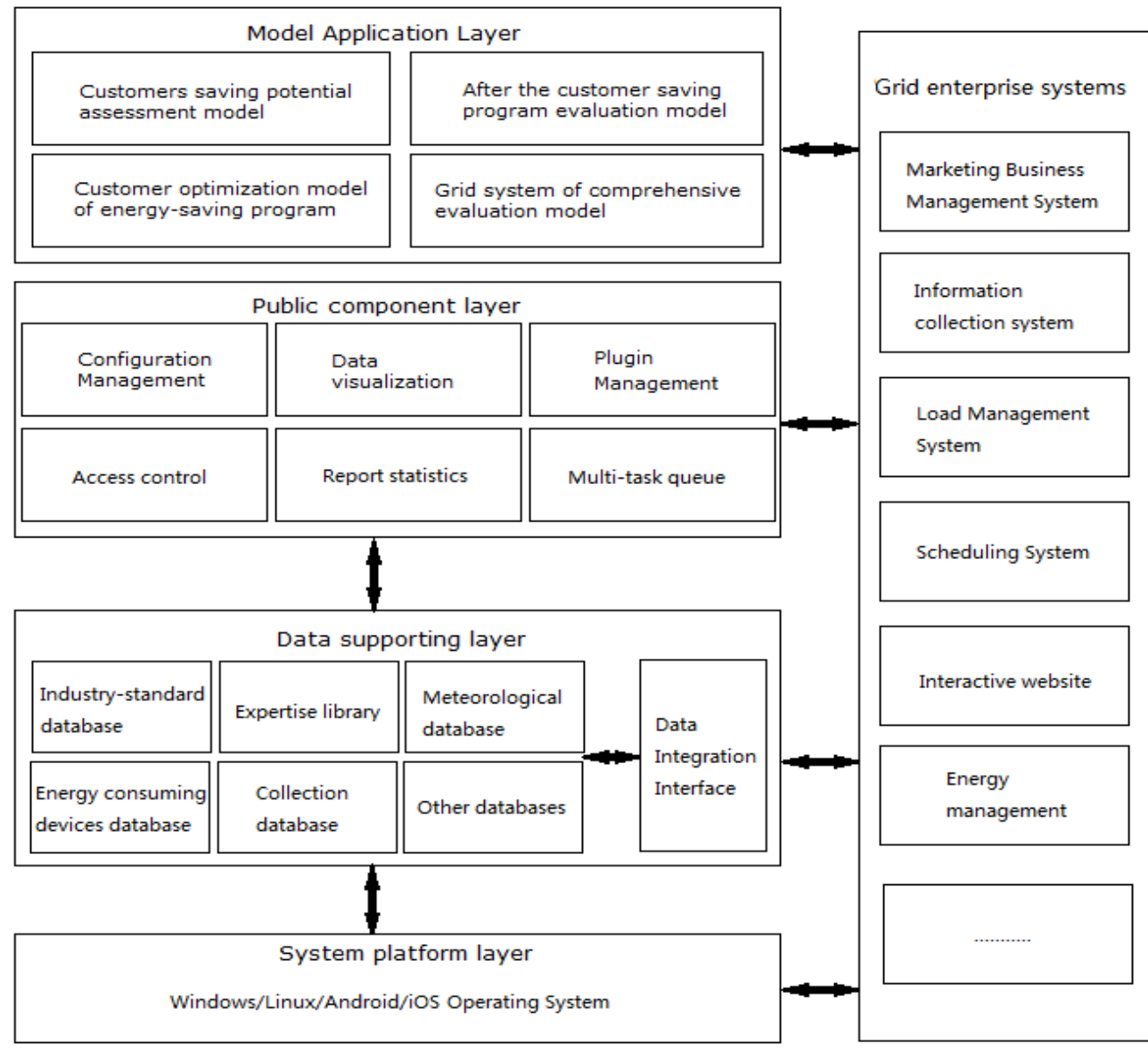

Figure 2. System Software Structure 
To adapt to different industries, different types of customer needs, development of the system to be compatible server platforms Windows, Linus, Android, ios and other mainstream operating systems, especially mobile terminal operating system supports cross-platform and mixed-platform operation. To achieve the client's system via mobile devices to remotely monitor energy use scenarios and focus adjustment state of the sensor by switching the scene fast mode. In addition, through interactive visualization techniques, so that different business data customization and browse, can be found in front of the system to show the graphical statistical reports.

Data supporting layer mainly provides general network communication support platform and real-time, historical multidimensional data management, network communications platform should be based on standard networking protocols TCP / IP, heterogeneous data networks in order to improve communication and heterogeneous operating system platform for data exchange system reliability. On the one hand by collecting raw data, centralized management of multiple systems with energy scenarios and terminal equipment, statistical information on equipment energy use, real-time fault detection data. On the other hand, on the basis of the establishment of long-term industry standard database, and evaluation expertise library database, and provide a uniform data transfer interface, database access interface and the control command interface via the data conversion component and the enterprise service bus and the external other systems for data exchange. Taking into account the heterogeneity of belonging is particularly obvious pattern is relatively dilute, low transaction requirements, in addition to the underlying database using the traditional RDBMS, the introduction of NoSQL solutions may be appropriate as a supplement, such as MongoDB, so as to accommodate a flexible data model and widespread across the Table, and even cross-query the database.

JSON data exchange using an uniform format information. And, like XML, JSON is based on the data in plain text format. Because JSON is JavaScript born ready, so, JSON data format is very simple and can be transmitted by a simple JSON String, Number, Boolean, you can transfer an array, or a complex Object object. As a lightweight data interchange format, JSON enough to express all kinds of information and data supporting layer database data objects.

Data integration interfaces in addition to reading and writing of data storage formats and encapsulation, but also provided between data objects between data objects and energy scenarios, a variety of interactive data conversion interface between the object and the actual participants, with based event-driven processing, based on a service-oriented processing, and multi-participant roles of terminal visual processing. Integrated interface via Web Service approach to deployment, the specific can be implemented as a Thrift Server. Full use of the Thrift framework C++, Java, Python, PHP and other languages of communication integration and support, and service implementation using these interfaces and interaction mechanisms upper internal logic of each subsystem, support custom development platform.

Public component layer in the support layer on the basis of the data to achieve a dataprocessing, event services, plug-in management, reporting services, graphics platform, system management, access services. The common abstraction of all kinds of industrial applications, providing a unified component interfaces, shared by the various application systems to enhance reusability of code, but also for systems development and maintenance convenience. Foreign public assemblies are provided in the form of Web Service, convenient and flexible deployment, once the component is running, its version number, as well as all the services can be provided through the platform layer query, and components are provided inheritable interface, running on their own dynamic environment perception, application services can intelligently perceive environments, registration up service they can offer, or query the existence of a dynamic environment and be available for service calls. 
Service registration and call public assemblies using RPC (Remote Procedure Call) approach, the client request by the parameterized encapsulated into an array or object, while receiving the same type of server responses. Request consists method/parameter/id three properties, the response also consists result/error/id three properties. A matching request and response process id interact with each other. Methid need globally unique within the component, methid between different components of the space by name to avoid naming conflicts.

Model application layer is to establish energy-efficiency model library on the basis of the data supporting layer, and public assembly layer on and power efficiency targets management, customer energy efficiency assessment, energy efficiency optimization, energy efficiency after the use of data mining, machine learning and other technology to customers and grid enterprises assessment services and other functions. Energy efficiency model library includes customer saving potential assessment model, the customer saving program efficiency optimization model of energy efficiency model grid companies three parts.

The basic attributes based on customer classification, electricity behavioral analysis, consumption pattern analysis, combined with expert experience and industry benchmarking data to assess the level of energy saving and energy-saving potential customers through the decision tree model (Decision Tree).

Based on the results of energy-saving potential customers evaluate candidate generation energy-optimized solutions, through energy benefits, costs, environmental impacts, risks, and many other indicators to determine the energy-optimized solutions for customer benefits.

Through the implementation of energy conservation programs for some time, the customer's energy efficiency targets periodic tracking assessments and joint evaluation report provides third-party evaluation agencies. Predict and assess the energy efficiency of the power grid and energy efficiency targets completion of the program impact on the power grid. Assessment of energy-saving programs for individual customers candidate gains the power grid, resulting in the distribution network customer base candidate energy-saving programs, consider multi-client interaction, combined with a comprehensive assessment of the grid system of energy-saving programs for customers from the power grid reliability, security, economic and other multi-angle earnings power grid, resulting in global optimization solution.

\subsection{System Business Process}

The system is mainly business-oriented energy conservation services, business objects into client module and the power grid enterprises module, the system implementation process shown in Figure 3. 


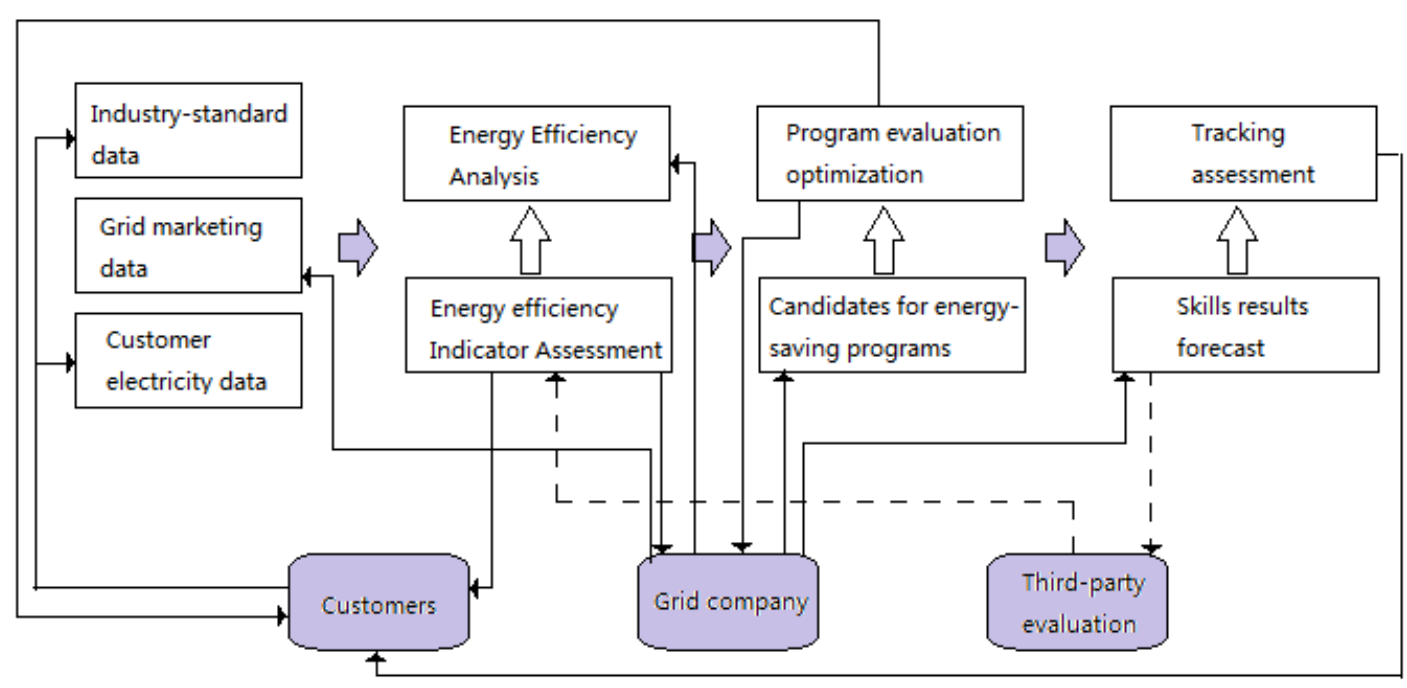

Figure 3. Systems Business Process

Energy efficiency data include: customer-owned industry benchmarking data, various electricity customer site data collection, internal marketing data grid enterprises, focusing on energy device file management data.

By index system of customer energy consumption statistics, comparing the same period, horizontal contrast, energy efficiency and provide a benchmark to compare the value of the industry with a third party evaluation agencies, clients come to the level of energy efficiency and the level of the same industry in the field; again saving potential through customer evaluation model reasoning, analysis and evaluation of energy-saving potential of the enterprise, thus helping customized energy efficiency goals.

Based on the results of reasoning saving potential customer evaluation model, resulting in energy-saving programs candidate, by saving benefits, costs, environmental impacts, risks, and many other indicators of energy-saving programs to build customer optimization model to assess the candidate inefficient energy-saving solutions for customers with the best level of energy efficiency assessment and recommendations, and determine energy-saving programs for customers benefits.

Energy efficiency monitoring platform for customers to show real-time and historical data, monitor customer site energy system operating conditions, the various parts of the energy consumption in real-time statistical units and rates; detect faults, real-time status of alarm limit alarm; real-time assessment of customer energy efficiency targets completion, thus achieving two-way interaction between the intelligent grid companies and customers.

Not only that, the platform also enables the customer site energy consumption monitoring, energy statistics, energy consumption analysis, focusing on energy equipment management, energy metering and other features that make the customer unit managers to grasp the proportion of the cost per unit of energy, trends, develop energy efficiency programs to all sectors, making clear the responsibility of energy conservation, and promote the healthy and stable development.

In addition, energy efficiency can also establish monitoring platform for customers and institutions to improve the management level, the realization of intelligent energy management systems, making routine maintenance easier, reducing the daily management costs per unit of energy systems clear, transparent, improve energy system efficiency and improve economic efficiency, saving energy costs.

Through a period of time the customer site energy efficiency monitoring and conservation programs specific implementation, the use of customer energy efficiency 
program after assessment model can be phased track assessment of the customer's energy efficiency goals, and comprehensive consideration of the grid side of the customer's actual energy efficiency gains were computing, managers and decision makers to provide some help, and joint evaluation report provides third-party evaluation agencies.

Through the establishment of the grid system of comprehensive evaluation model, and this year has signed various types of energy service customers evaluate energy efficiency to help power companies predict and evaluate energy-saving targets completion; and in combination with customer-centric analysis and complex situations comprehensive grid system assessment of energy-saving programs, scientific and effective way to build customer and grid energy-smart decision-making system, to provide specific recommendations for energy-saving power companies and their customers.

\section{The Energy Efficiency of the Application Management System}

\subsection{Power Collection System}

Power collection management automation system (power system) is set to automatically collect electricity, transport, statistics, analysis, settlement in one of the automated system, the power to implement commercial operation and management of electricity to the market technical support, the full realization of hair, transmission and distribution of electric energy grid users automated collection, analysis and measurement functions such as automation systems. However, the current situation of power enterprise computer application point of view, the various information systems within the enterprise relatively independent, forming many islands of data, it is difficult to realize the power management support for the entire process. Therefore, to meet the electric remote metering of energy and its commercial operation of integrated application systems need to build a power-based automatic acquisition is very necessary.

Electricity acquisition system uses a hierarchical, distributed architecture, from the main station, substation electricity collection terminals, distribution transformer, large user acquisition terminal and communication networks. Structure of the main station for distributed local area network, the data acquisition system by configuring different communication modules to network, analog or digital line / dial, Global System for Mobile Communications (Global System for Mobile Communications, GSM), General Packet Radio Service (General Packet Radio communicate Service, GPRS) or optical fiber and collection terminals, while transcribing station end information collection device. Data transfer support master mode and terminal active call reporting mode, the terminal will power and time stamp data uploaded to the master station. Master can send broadcast commands, while the completion of the terminal unification of energy meter data freeze, power meter processor reset functions.

Electricity substation power collection terminal including terminal, distribution transformers and large user acquisition terminal, as is shown in Figure 4. The main function is to collect the data stored energy meter monitoring system operating conditions, in accordance with a predetermined communication protocol to exchange information with the master station through different means of communication. 


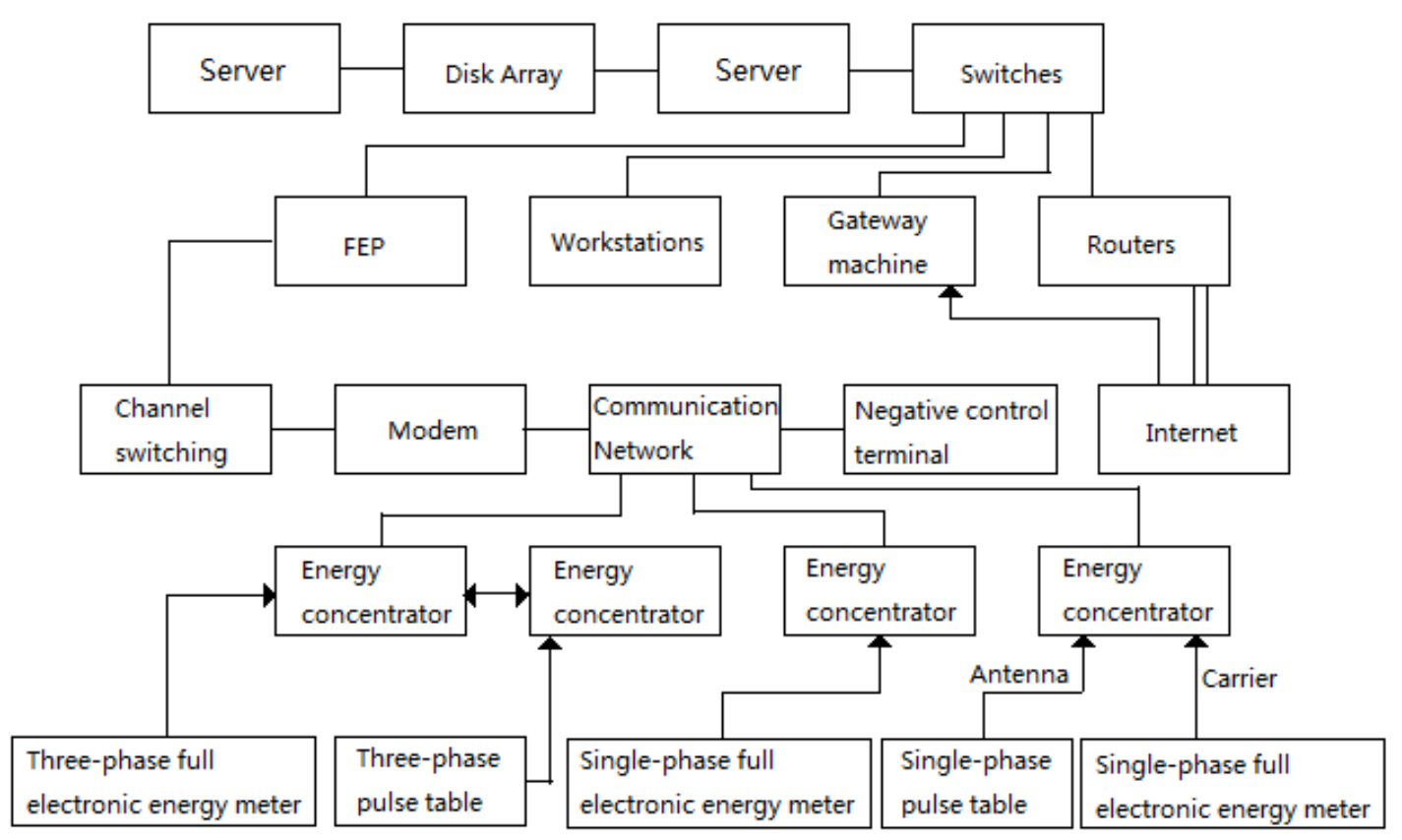

Figure 4. Power Remote Metering Management System Diagram

\subsection{Customer Payment System}

Depending on the purpose of the power to develop a variety of fees and charges of the way, the main types are: residents object to household electricity; to office buildings, shops and other business electricity as an object; targeting high-voltage industrial and Special high-voltage and other large-scale industrial enterprises use. Improve electricity customers a variety of payment methods for population characteristics of different electricity customers' purchase difficult "situation, as far as possible to provide customers a variety of payment channels, create a harmonious environment charges, lower electricity cost recovery, in order to make power companies service work in harmony to improve and develop in harmony.

Which charges modes include: operating room charge mode; bank collecting electricity mode; walk closed mode; electricity prepaid card payment mode; agents charging mode; online business hall-powered charging mode.

The province's centralized database networked charging mode. Construction provincial capital recovery and centralized model of electricity management system is to achieve intensive, meticulous management needs, but also the first step in a large centralized mode power marketing system construction. With the implementation of the State Grid Corporation SG186 project, centralized management model represents a trend in the direction of power supply enterprise marketing MIS construction and development at all levels, is a more advanced technology. After charging the implementation of a centralized database, the user can in the province of any one region in the province and even the domestic payments, so you can easily achieve remote payments, will facilitate the implementation of prepaid cards, online banking and other payment modes. Meanwhile, the province's data centralization carried directly to the power supply enterprise to bring the following benefits: After the hand dataset, electricity can be received directly accounted for, so that you do not have a level of up to allocate, effectively shortening the retention time funds; electricity on the other hand received directly accounted for, would also facilitate the timely completion of the electricity supply companies paid tasks.

The overall procedure municipal electricity supply companies to build centralized management mechanism to be divided into two parts, the first step to set up and relevant organizations locate major electricity centralized management; second step, to strengthen 
the centralized control from the perspective of the copy table review, electricity accounting, fund collection and accounts processing, real-time inspection and collection of electricity monitoring, assessment and training and other business sectors and to sort out the specific operational procedures, the implementation of intensive management, thus forming the municipal electricity supply enterprises centralized management mechanisms.

In accordance with the idea of centralized control, electricity centralized management mechanism includes the following organizations:

a. Electricity accounting center-electricity centralized management hub body. Responsible for reviewing the city's electricity business guidance and supervision and evaluation, through all levels of the electricity business, all kinds of work, link, link up dynamic elements to achieve electricity intensive business management and flat management.

b. Counties RC electricity management department-connecting electricity powered by accounting center and primary business ties. Responsible for auditing the wrong tariff accounting center to track electricity tariff treatment. Urge the power supply business for verification, and is responsible for feedback related to the verification report; implementation of professional management of power and charges the meter reading business.

c. The power supply business-copy, nuclear, closing specific business managers who. Meter reading, reminder fees and charges responsible for electricity customers, the review found that electricity customers with customer information and payment anomalies, with electricity accounting centers and county departments for review RC electricity users in the region under the jurisdiction of the volumes and meter reading planning work.

d. Council Finance Department-electricity fund account, fund collection and paid billing manager. Responsible for managing the global tariff revenue bank accounts; responsible for the financial accounting of funds paid-tariff offered by banks fetched notice; responsible for corporate banking journal check with the bank (electronic) reconciliation data.

e. Financial institutions-electricity funds collection, transfer and paid reconciliations important auxiliary bodies. Implementation of centralized management and control of electricity. In terms of the recovery of funds and timely transfer, in terms of loss of funds reconciliation. Require the cooperation and support of financial institutions.

f. The information sector-electricity centralized accounting system maintenance and technical support organizations. Centralized accounting-related charge of electricity system operation and silver electric networked systems maintenance, data backup, to ensure safe and stable operation.

\subsection{Power Management and Customer Service System}

Functional customer support system can be grouped into three areas: a) for sales, marketing and customer service business processes into three parts information: b) with customers means (such as telephone, fax, Internet, Email and other communication required to be) integration and automation; c) information processing function of the above two parts were accumulated, resulting in intelligent customer analysis, corporate strategy and tactical decisions for support. The implementation of the requirements of power supply enterprise information platform will not only pay attention to the development of new customers, but also to focus on keeping existing customers and potential excavation. Through a comprehensive recording and analysis of customer contacts, deepening understanding of customer needs, develop the potential of existing customers to purchase exists, to further increase sales, reduce costs, increase profitability and improve customer satisfaction degree target.

On the basis of the integration platform, to carry out basic production building business applications; achieve interconnection and marketing systems, providing support and 
protection for grid interconnection and user information; implementation and interconnected financial system, to provide support for the integration of production equipment construction equipment assets.

a. Adhere to the platform of ideas, building integrated grid platform. To achieve the integration of data network equipment to support the city grid network topology, geographic information, and by a variety of supply side and user side collection systems such as real-time data acquisition.

b. Through the integration of grid platform construction, lose change with model integrated design. And business support through a unified platform to achieve grid model integration, grid integration and network topology integrated graphics.

c. To achieve a variety of productive integration of business operations and conduct business with the integrated grid platform, production and business management systems with business data and processes cascade closed-loop management.

d. Achieved through vertical and horizontal integration. By construction, and financial systems integration interconnected grid platform, providing support for the integration of production equipment construction equipment assets; through the construction of integrated grid platform and marketing system interconnection, power and user information to achieve interconnection support.

e. Give full consideration to the use of sophisticated software packages.

\subsection{Regulatory System}

Electrical safety supervision is to ensure the safe operation of the power grid, power companies efficient production, the basic work of the country's energy security. Establish an advanced computer management system is to achieve electrical safety supervision work efficiently and effectively manage the inevitable choice. Currently, the province each power company has professional management system for safety supervision, but generally scattered in various subsystems, do not form an orderly plan of professional systems, resulting in a lack of coherence in the work process, unity, safety supervision work difficult to achieve closed-loop management. Therefore, it is extremely necessary to previously decentralized, non-uniform safety supervision system in a unified form of professional string together to achieve a task manager, a complete closed-loop system of work safety supervision work, making safety supervision and management of energy efficient, continuous conducted.

In order to ensure electrical equipment is in good working condition and excellent performance, the need of scientific and strict management and supervision of a complete set of technical means, technical supervision has become an important part of the power industry management. Technical supervision and management of electricity can be divided into 10 special called ten supervision, namely: metal supervision, chemical supervision, supervision insulation, thermal supervision, supervision of electrical measurement, power quality supervision, environmental monitoring, protection supervision, energy conservation supervision, measurement and supervision. Which the power supply enterprises are more important and common chemical supervision, (high pressure) insulation supervision, protection and supervision three, called the three oversight, in this system primarily directed at these three supervisory management.

Business management and technical supervision of the object is equipment testing, would have required all aspects of equipment throughout the trial, including the development of test plans, the actual operation of the test methods and procedures, test results, test summary, etc., but the specific implementation in the system, specific work equipment tests conducted by other subsystems to production management subsystem implementation, management subsystem in production technology and technical supervision module is invoked test reports from other subsystems query function, designed for technical supervision and technical supervision work produced a summary report and report to management. 
Technical supervision is the basis of test data and test a variety of devices conclusions based on the data generated. Among them, the main source of data is the oil works supervision chemical tests (such as chromatography, etc.), the degree of pollution analytical chemistry test (salt analysis, etc.), the main source of data is the high voltage insulation monitoring test, the main source of the data protection supervision is checking secondary equipment. The main source of data is the measurement of metrological supervision and management subsystem measurement of five rate (pass rate, parity rate, rotation rate, accuracy rate, the failure rate).

\section{Conclusion}

In this paper, combined with the integration of energy efficiency business management system has been constructed successfully, and to the distribution network GIS system is the foundation and core focus on integrated marketing and management information systems, with a net production of management information systems, distribution network project management information system, metering automation systems, according to actual situation gradually integrated distribution network planning systems, scheduling automation systems, data distribution network automation system, required to achieve customer-oriented supply reliability management, auxiliary fault repair, business expanding supply scenario analysis, engineering data electronically transfer line loss "four" management, distribution network planning decisions, customer business with comprehensive information queries, assist the development of meter reading routes, near real-time data display and other business functions. The basic purpose of the energy center management system is to improve the energy system in operation, management efficiency, energy consumption in the production process to find the best process data, providing a proven, effective, easy to use and control the overall energy system solutions ; set of advanced, reliable and secure energy system operation, operation and management platform. And to achieve security and stability, economic balance, the basic objectives of quality and environmental protection, supervision and evaluation.

\section{References}

[1] J. M. G. Fornieles, I. S. Fan and A. Peter, “A Work Breakdown Structure that Integrates Different Views in Aircraft Modification Project", Concurrent Engineering: Research and Application, vol. 11, no. 47, (2003).

[2] M. Y. Leung, Y. S. Chan and O. Paul, "Impact of Stress on the Performance of Construction Project Managers", Journal of Construction Engineering and Management, vol. 134, no. 644, (2008).

[3] J. C. Paul, D. Y. Alisha and R U. Dawn, "A Preliminary Assessment of the Relationship between Project Success, System Engineering, Team Organization”, Engineering Management Journal, vol. 20, no. 40, (2008).

[4] S. C. Andrew, "Reasons for Cost and Schedule Increase for Engineering Design Projects", Journal of Management in Engineering, vol. 18, no. 29, (2002).

[5] T. A. Frank, G. C. Elias and J. V. Robert, "Post-project Reviews as a Key Project Management Competenc", Technovation, vol. 28, no. 633, (2008).

[6] E. Basil, "Evaluating Development Assistance: A Review of the Literature", Public Administration and Development, vol. 8, no. 75, (1988).

[7] D. R. Gines, "Cost-Benefit Analysis of the High-Speed Train in Spain", The Annals of Regional Science, vol. 31, no. 175, (1997).

[8] L. K. Ralph, "Utility Functions for Equity and Public Risk", Management Science, vol. 26, no. 345, (1980).

[9] M. Christoph, D. De and W. Rik, "LCC Analysis of Different Resonant Cireuits and Solid-State Circuit Breakers for Medium-Voltage Grids", IEEE Transaetions on power Delivery, vol. 21, no. 1414, (2006).

[10] T. Shimakage, K. Wu, T. Kato, T. Okamoto and Y. Suzuoki, "Life-Cycle-Cost Comparison of Different Degradation Diagnosis Methods for Cables”, Electrieal Insulation Materials, vol. 3, no. 737, (2005). 


\begin{abstract}
Author
Hu Bo, He received the B.S. and M.S. degrees in computer and application from North China Electric Power University, Beijing, China, in 1995 and 2006, respectively, and the Ph.D. degree in power system and automation from the North China Electric Power University, Beijing, China, in 2013.

$\mathrm{He}$ is a senior engineer in the State Grid Liaoning Electric Power Co. Ltd. He has participated in many important national technological projects and gotten great efforts, which made him honored the national science and technology progress award once and provincial and ministerial level 5 times. His research interests include complex networked systems including smart power grid, planning and scheduling of electrical power and electric power markets.
\end{abstract}

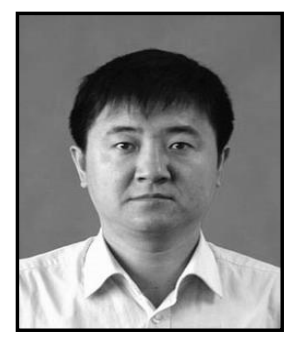

